\title{
USO DE CONJUNTOS TERMINAIS PARAMETRIZADOS PARA TRATAMENTO DE PROBLEMAS DE NÃO-FACTIBILIDADE EM CONTROLE PREDITIVO
}

\author{
Rubens Junqueira Magalhães Afonso* \\ rubens jmeita.br
}

\author{
Roberto Kawakami Harrop Galvão* \\ kawakami@ita.br
}

*Instituto Tecnológico de Aeronáutica, Divisão de Engenharia Eletrônica, 12228-900 São José dos Campos, SP, Brasil

\begin{abstract}
Employment of Parametrized Terminal Sets for Infeasibility Handling in Predictive Control

Terminal constraints are usually employed in predictive control formulations to provide closed-loop stability guarantees. However, such guarantees are lost if the associated optimization problem is not feasible from the beginning or if it becomes infeasible due to the onset of faults, for example. This work presents an approach involving setpoint management and relaxation of operational constraints to address infeasibility problems in predictive control. It is assumed that infeasibility may occur either at the beginning of the control task, due to an adverse initial condition, or as the result of an actuator fault that reduces the range of admissible control values. The proposed approach involves the parametrization of the maximal output admissible set (MAS) employed as terminal constraint in the control law. This parametrization avoids the need to repeat the MAS determination during the infeasibility handling procedure. For illustration purposes, a case study involving a simulation model is presented.
\end{abstract}

KEYWORDS: Predictive control, Constraints, Feasibility, Setpoint management.

\section{RESUMO}

Restrições terminais são tipicamente empregadas em formulações de controle preditivo para garantia de estabilidade em malha fechada. Contudo, tal garantia é perdida caso o problema de otimização associado não seja factível no instante inicial ou deixe de ser factível devido, por exemplo, à ocorrência de falhas. Este trabalho apresenta uma abordagem de gerenciamento de referência aliado ao relaxamento de restrições operacionais como forma de tratar problemas de nãofactibilidade em controle preditivo. Considera-se que a nãofactibilidade pode ocorrer tanto no início da tarefa de controle, em decorrência de uma condição inicial desfavorável, como pode ser causada por uma falha de atuador que reduza a faixa de valores de controle permitidos. A abordagem proposta envolve a parametrização do conjunto máximo admissível de saída (MAS) utilizado como restrição terminal na lei de controle. Evita-se assim a necessidade de se repetir a determinação do MAS durante o tratamento da nãofactibilidade. Para fins de exemplo, apresenta-se um estudo de caso com um modelo de simulação.

PALAVRAS-CHAVE: Controle preditivo, Restrições, Factibilidade, Gerenciamento de referência. 


\section{INTRODUÇÃO}

O termo Controle Preditivo Baseado em Modelo (Modelbased Predictive Control, MPC) denomina uma ampla gama de técnicas que empregam realimentação dos sensores da planta a cada período de amostragem para calcular a solução de um problema de controle ótimo em um horizonte móvel (Normey-Rico, 2007), (Camacho e Bordons, 1999). Originalmente, o desenvolvimento de tais técnicas foi motivado por aplicações na indústria de petróleo (Cutler e Ramaker, 1980). Entretanto, outros setores vêm cada vez mais se beneficiando do emprego de abordagens de MPC (Qin e Badgwell, 2003). Com efeito, trabalhos recentes relatam que esta estratégia pode ser empregada com sucesso também em aplicações de dinâmica rápida, tais como as encontradas no setor aeronáutico, por exemplo (Keviczky e Balas, 2006), (Lopes, 2007), (Almeida e Leissling, 2010). Uma das principais vantagens do MPC consiste na capacidade de tratar restrições nas entradas e estados da planta, possibilitando a obtenção de ganhos de desempenho e maior segurança de operação (Maciejowski, 2002), (Rossiter, 2003). Em particular, restrições sobre a excursão dos sinais de controle são comuns em processos que operam perto das suas condições economicamente ótimas (Rodrigues e Odloak, 2005).

A estabilidade da malha de controle com o MPC pode ser garantida por meio do uso de horizonte de predição infinito ou através da introdução de restrições terminais (Maciejowski, 2002), (Rossiter, 2003). Neste último caso, pode-se impor que o estado atinja o ponto de equilíbrio desejado ao final do horizonte de predição ou, de forma menos restrita, que ingresse em um determinado conjunto terminal que seja invariante (Gilbert e Tan, 1991) sob a ação de um controlador local estabilizante. Contudo, para que as garantias de estabilidade sejam preservadas, o problema de otimização deve ser factível a cada instante de amostragem. Neste contexto, torna-se imperativo o desenvolvimento de técnicas de recuperação da factibilidade, caso o problema não seja factível no instante inicial de operação ou deixe de ser factível, por exemplo, devido à ocorrência de uma falha.

Uma solução simples para a recuperação da factibilidade consiste no aumento do horizonte utilizado, de forma a proporcionar mais graus de liberdade para a otimização. No entanto, aumenta-se, por consequência, a carga computacional envolvida, visto que o problema passará a envolver um número maior de variáveis de decisão.

Como alternativa, pode-se recorrer a estratégias de relaxamento de restrições. Segundo (Vada, Slupphaug e Johansen, 2001), as restrições a serem tratadas por um MPC podem ser divididas nas seguintes classes:
- Restrições físicas: São restrições que devem ser respeitadas durante toda a operação. Os limites de operação de uma válvula, que pode estar aberta entre 0 e $100 \%$ de sua capacidade, constituem um exemplo de restrições físicas.

- Restrições operacionais: São restrições que se deseja respeitar a fim de obter um comportamento mais adequado, mas que podem ser relaxadas caso ocorra nãofactibilidade. Por exemplo, pode ser vantajoso operar um reator químico em uma determinada faixa de temperaturas, de forma a acelerar a formação de produtos de interesse econômico. Entretanto, no caso de ser impossível que isso seja feito sem comprometer a segurança de operação da planta, estas restrições podem ser relaxadas.

A literatura é vasta em diferentes abordagens para realizar o relaxamento das restrições. Rawlings e Muske (1993) sugerem remover as restrições durante um período inicial do horizonte de predição garantindo a factibilidade da otimização. Após esse período, as restrições voltam a ser consideradas. Zafiriou e Chiou (1993) propõem uma forma de calcular a magnitude do relaxamento necessário para tornar o problema de otimização factível, considerando um sistema de entrada e saída únicas. O trabalho de Scokaert (1994) contém diversas propostas para contornar o problema da não-factibilidade, entre elas, uma abordagem que consiste em classificar as restrições em níveis de prioridade e satisfazer as de níveis mais altos, relaxando as de níveis mais baixos. Scokaert e Rawlings (1996) apresentam uma abordagem capaz de minimizar a duração e o pico das violações das restrições impostas ao sistema, melhorando as características da resposta transiente. No que tange ao relaxamento de restrições tanto sobre o sinal de controle quanto sobre as saídas, uma técnica encontra-se descrita em (Alvarez e de Prada, 1997). Critérios de prioridades previamente definidos são usados no relaxamento da amplitude ou da taxa de variação dos sinais de controle. Já as restrições de saída são relaxadas na amplitude ou no intervalo de duração ao longo do horizonte de predição. Em (Vada, Slupphaug, Johansen e Foss, 2001) a solução proposta consiste em classificar as restrições em níveis de prioridades e adicionar um problema de programação linear para ser resolvido em paralelo com o problema de otimização padrão do MPC. Uma revisão dessas diversas técnicas é apresentada em (Afonso e Galvão, 2012) com exemplos de aplicação. No âmbito de acomodação de falhas de atuador, Afonso e Galvão (2010) trataram o problema de priorização de restrições através da agregação de diferentes variáveis de folga às restrições de saída da planta. O menor relaxamento necessário era então obtido através da solução de um problema de programação linear, atribuindo-se pesos às variáveis de folga de acordo com a prioridade de cada restrição. 
Outra forma de recuperar a factibilidade são os chamados procedimentos de "gerenciamento de referência" (Bemporad e Mosca, 1994), (Gilbert e Kolmanovsky, 1995), (Bemporad et al., 1997), que reduzem artificialmente a distância entre o estado da planta e o conjunto terminal de restrições. O reference governor proposto por (Kapasouris et al., 1988) inspira diversas técnicas de manipulação do sinal de referência ou do erro de rastreamento para lidar com problemas em que há saturação dos atuadores (Gilbert e Kolmanovsky, 1995). Em (Afonso e Galvão, 2010b) utiliza-se uma dessas técnicas para tratamento de não-factibilidades advindas de falhas de atuador em um modelo de helicóptero de laboratório. Há também trabalhos visando impor um comportamento à planta por meio de um modelo de referência que se utilizam da introdução de uma variável de relaxamento para obter factibilidade quando existem limitações dos sinais de controle (Montandon et al., 2008).

As garantias de estabilidade em abordagens de gerenciamento de referência podem ser mantidas por meio do emprego de conjuntos terminais invariantes previamente determinados considerando a possibilidade de mudança na referência. Limon et al. (2008) usam uma abordagem desse tipo, parametrizando o conjunto terminal em termos dos valores de equilíbrio tanto do controle como do estado. Os autores mostram que um gerenciamento ótimo da referência pode ser feito de forma a garantir o menor afastamento possível entre o valor de equilíbrio desejado e o usado pelo MPC, mantendo a factibilidade. Como resultado, é possível aumentar o domínio de atração proporcionado pelo controlador.

Uma aplicação da parametrização do conjunto terminal em termos do valor estacionário do controle pode ser encontrada em (Almeida e Leissling, 2010). Nesse trabalho, os procedimentos utilizados para parametrização e obtenção de um valor ótimo de regime são similares aos adotados em (Limon et al., 2008). Contudo, a aplicação é estendida para tratar casos de não-factibilidade advinda de falhas de atuador. No caso de uma falha desse tipo, dispondo-se de informação sobre sua localização e magnitude, pode-se reconfigurar o MPC de forma a levar em conta as novas condições dos atuadores. Em particular, falhas que reduzam a faixa de operação do atuador podem ser tratadas pelo MPC por meio da mudança das restrições impostas sobre a excursão do sinal de controle. No entanto, torna-se necessário recorrer à parametrização do conjunto terminal de restrições em termos também dos valores possíveis de restrições sobre os controles, a fim de evitar a necessidade da redeterminação dos mesmos. Com efeito, em aplicações de dinâmica rápida, a complexidade dos cálculos envolvidos na determinação do conjunto invariante podem impossibilitar a sua atualização em tempo real.

No presente trabalho, recorre-se a uma estratégia de parametrização do conjunto terminal invariante em termos dos possíveis valores de equilíbrio das variáveis de controle e estado, bem como das restrições impostas sobre elas. Dessa forma, torna-se possível reconfigurar o conjunto terminal em tempo real quando o gerenciamento de referência ou o relaxamento de restrições se fizerem necessários a fim de recuperar a factibilidade do problema de otimização. Pode-se ainda reconfigurar o MPC com os novos valores de restrições impostas sobre o controle em decorrência da falha. Por considerar a parametrização em termos tanto de valores de equilíbrio quanto das restrições de excursão das variáveis de controle e estados, este trabalho traz contribuições em relação a (Limon et al., 2008) e (Almeida e Leissling, 2010). A estratégia proposta é ilustrada por meio de um estudo de caso simulado, considerando tanto o problema de não-factibilidade inicial quanto a ocorrência de falha de atuador.

$\mathrm{O}$ restante do texto encontra-se estruturado da seguinte forma. A Seção 2 detalha a formulação de MPC adotada neste trabalho. A Seção 3 apresenta as técnicas de gerenciamento de referência e relaxamento da restrições, assim como a parametrização proposta para o conjunto terminal. Resultados de simulação são discutidos na Seção 4. Finalmente, a Seção 5 traz as conclusões obtidas, bem como sugestões de trabalhos futuros.

\subsection{Notação}

$\diamond x_{P} \in \mathbb{R}^{n}:$ estado da planta;

$\diamond x_{r e f} \in \mathbb{R}^{n}$ : valor desejado para o estado;

$\diamond x=x_{P}-x_{\text {ref }}$;

$\diamond \mu \in \mathbb{R}^{n}$ : estado fictício de equilíbrio após o gerenciamento de referência;

$\diamond u_{P} \in \mathbb{R}^{p}$ : controle aplicado à planta;

$\diamond u_{\text {ref }} \in \mathbb{R}^{p}$ : controle em regime associado a $x_{\text {ref }}$;

$\diamond \eta \in \mathbb{R}^{p}$ : valor de controle de equilíbrio fictício associado a $\mu$;

$\diamond v \in \mathbb{R}^{p}$ : controle gerado pelo MPC;

$\diamond u \in \mathbb{R}^{p}$ : controle gerado pelo MPC adicionado ao da malha interna;

$\diamond y_{P, \max } \in \mathbb{R}^{q}$ : restrição operacional superior da saída;

$\diamond y_{P, \min } \in \mathbb{R}^{q}$ : restrição operacional inferior da saída;

$\diamond y_{P, \max }^{f i s} \in \mathbb{R}^{q}$ : restrição física superior da saída;

$\diamond y_{P, \min }^{f i s} \in \mathbb{R}^{q}$ : restrição física inferior da saída;

$\diamond u_{P, \max } \in \mathbb{R}^{p}$ : restrição superior do controle em condições normais de operação;

$\diamond u_{P, \min } \in \mathbb{R}^{p}$ : restrição inferior do controle em condições normais de operação;

$\diamond u_{f a l, \max } \in \mathbb{R}^{p}$ : restrição superior de controle após a falha de atuador;

$\diamond u_{\text {fal,min }} \in \mathbb{R}^{p}$ : restrição inferior de controle após a falha de atuador;

$\diamond \epsilon_{s} \in \mathbb{R}^{q}$ : variável de folga associada ao relaxamento da restrição superior de saída; 


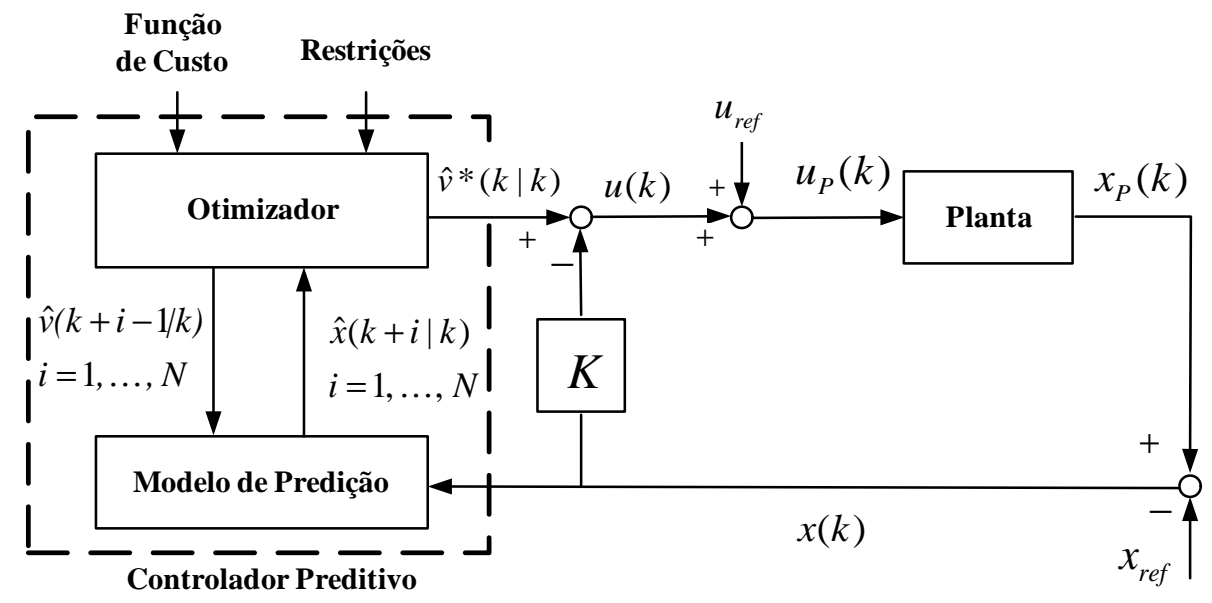

Figura 1: Controle preditivo com malha interna.

$\diamond \epsilon_{i} \in \mathbb{R}^{q}$ : variável de folga associada ao relaxamento da restrição inferior de saída;

$\diamond k$ : instante atual;

$\diamond \hat{\bullet}(k+i \mid k)$ : valor previsto da variável $\bullet$ no instante $k+i$ com base nas informações disponíveis até o instante $k$;

$\diamond \bullet *$ : valor ótimo da variável •;

$\diamond N \in \mathbb{N}$ : horizonte do MPC;

$\diamond K \in \mathbb{R}^{p \times n}$ : ganho da malha interna;

$\diamond I_{n}$ : matriz identidade de dimensão $n \times n$;

\section{FORMULAÇÃO DE CONTROLE PREDI- TIVO ADOTADA}

A Fig. 1 apresenta os principais elementos da formulação de MPC usada neste trabalho. Como a implementação está na forma de regulador, o ponto de equilíbrio $x_{r e f}$ desejado para o estado deve ser subtraído do estado medido da planta $x_{P}$, de modo a gerar o estado $x$ lido pelo controlador, isto é:

$$
x=x_{P}-x_{r e f} .
$$

De maneira similar, o valor de equilíbrio correspondente para o controle $\left(u_{r e f}\right)$ deve ser adicionado à saída do controlador $u$ para gerar o controle $u_{P}$ a ser aplicado à planta, ou seja:

$$
u=u_{P}-u_{r e f} .
$$

Assume-se que a dinâmica da planta seja descrita por uma equação de estado a tempo discreto da forma $x_{P}(k+1)=$ $A x_{P}(k)+B u_{P}(k)$. Desse modo, a relação entre $u$ e $x$ é dada por

$$
x(k+1)=A x(k)+B u(k) .
$$

Deseja-se projetar o controlador preditivo de modo a respeitar restrições do tipo

$$
u_{P, \min } \leq u_{P} \leq u_{P, \max },
$$

$$
y_{P, \min } \leq y_{P} \leq y_{P, \max },
$$

sendo $y_{P}$ relacionada com o estado da planta por uma equação de saída $y_{P}=C x_{P}$. Tendo em vista (1) e (2), as restrições (4) e (5) podem ser expressas como

$$
\begin{gathered}
u_{P, \min }-u_{r e f} \leq u \leq u_{P, \max }-u_{r e f}, \\
y_{P, \min }-C x_{r e f} \leq y \leq y_{P, \max }-C x_{r e f},
\end{gathered}
$$

sendo $y=C x$.

O problema de otimização a ser resolvido no instante $k$ consiste em minimizar uma função de custo da forma

$$
J_{m p c}=\sum_{i=0}^{N-1} \hat{v}^{T}(k+i \mid k) \Psi \hat{v}(k+i \mid k),
$$

sujeita às seguintes restrições:

$$
\begin{aligned}
& \hat{u}(k+i \mid k)=-K \hat{x}(k+i \mid k)+\hat{v}(k+i \mid k), i \geq 0 \\
& \hat{v}(k+i \mid k)=0, i \geq N \\
& \hat{x}(k+i+1 \mid k)=A \hat{x}(k+i \mid k)+B \hat{u}(k+i \mid k), i \geq 0 \\
& \hat{x}(k \mid k)=x(k) \\
& \hat{y}(k+i \mid k)=C \hat{x}(k+i \mid k), i \geq 0 \\
& \hat{u}(k+i \mid k) \in \mathbb{U}, i \geq 0 \\
& \hat{y}(k+i \mid k) \in \mathbb{Y}, i>0
\end{aligned}
$$

em que $\Psi=\Psi^{T}>0$ é uma matriz de peso e $\mathbb{U}$ e $\mathbb{Y}$ são respectivamente os conjuntos de controles e saídas admissíveis em vista de (6) e (7).

Seguindo uma política de horizonte retrocedente, o controle no instante $k$ é dado por $u(k)=\hat{v}^{*}(k \mid k)-K x(k)$, sendo $K$ 
o ganho da malha interna representada na Fig. 1. No instante $k+1$, a otimização é repetida de modo a obter $\hat{v}^{*}(k+1 \mid k+1)$.

O controlador da malha interna é projetado como um regulador linear-quadrático (linear-quadratic regulator, LQR) com a seguinte função de custo:

$$
\begin{gathered}
J_{l q r}=\sum_{i=0}^{\infty}\left[\hat{x}^{T}(k+i \mid k) Q_{l q r} \hat{x}(k+i \mid k)+\right. \\
\left.+\hat{u}^{T}(k+i \mid k) R_{l q r} \hat{u}(k+i \mid k)\right] \\
Q_{l q r}=Q_{l q r}^{T} \geq 0, \quad R_{l q r}=R_{l q r}^{T}>0
\end{gathered}
$$

sendo $Q_{l q r}$ escolhida de modo que o par $\left(A, Q_{l q r}^{\frac{1}{2}}\right)$ seja detectável.

Seja $P$ a única solução simétrica não-negativa da equação algébrica de Riccati $P=A^{T} P A-A^{T} P B\left(R_{l q r}+\right.$ $\left.B^{T} P B\right)^{-1} B^{T} P A+Q_{l q r}$. Pode-se então mostrar que, se a matriz de peso $\Psi$ for escolhida como $\Psi=R_{l q r}+B^{T} P B$, então a minimização do custo (8) sujeito a (9) - (15) equivale à minimização do custo (16) sujeito a (11) - (15) (Chisci et al., 2001). Tem-se então a vantagem de se trabalhar com uma função de custo com horizonte infinito, o que é útil para garantia de estabilidade. Essa formulação foi introduzida por (Scokaert e Rawlings, 1998) e posteriormente colocada na forma aqui adotada por (Kouvaritakis et al., 1998). Vale notar que, devido à penalização do sinal de controle $\hat{v}$ em (8), o MPC só atua quando é necessário corrigir o controle da malha interna a fim de evitar violação das restrições (14) e (15).

Definindo o vetor $\hat{V}$ e a matriz $\bar{\Psi}$ como

$$
\hat{V}=\left[\begin{array}{c}
\hat{v}(k \mid k) \\
\vdots \\
\hat{v}(k+N-1 \mid k)
\end{array}\right], \bar{\Psi}=\left[\begin{array}{ccc}
\Psi & \ldots & 0 \\
\vdots & \ddots & \vdots \\
0 & \ldots & \Psi
\end{array}\right]
$$

a função de custo pode ser reescrita como

$$
J_{m p c}=\hat{V}^{T} \bar{\Psi} \hat{V}
$$

que é quadrática em termos de $\hat{V}$.

Vale ressaltar que a presença de infinitas restrições em (14) e (15) impede o uso direto de métodos computacionais para solução do problema de otimização. Contudo, essa dificuldade pode ser contornada introduzindo-se uma restrição terminal para o estado na forma de um "Conjunto Máximo de Saída Admissível" (Maximal Output Admissible Set - MAS) (Gilbert e Tan, 1991), conforme definido no Apêndice A.

Com efeito, para $i \geq N$, tem-se uma lei de controle dada simplesmente pela realimentação de estado $\hat{u}(k+i \mid k)=$ $-K \hat{x}(k+i \mid k)$ (vide restrições (9) e (10)). Desse modo, a evolução do estado passa a ser descrita por uma equação nãoforçada da forma $\hat{x}(k+i+1 \mid k)=(A-B K) \hat{x}(k+i \mid k)$, sendo $(A-B K)$ uma matriz com autovalores no interior do círculo unitário. Portanto, tomando uma variável de saída $z$ definida como

$$
z=\left[\begin{array}{l}
y \\
u
\end{array}\right]=\left[\begin{array}{c}
C \\
-K
\end{array}\right] x
$$

pode-se empregar o procedimento descrito no Apêndice A para obter um MAS, denotado por $\mathbb{O}_{\infty}$, tal que as restrições (14) e (15) sejam equivalentes a impor $\hat{x}(k+N \mid k) \in \mathbb{O}_{\infty}$. Ainda como discutido no Apêndice A, tal restrição terminal equivale a impor as restrições (14) e (15) até $i=N+t^{*}$, sendo $t^{*}$ obtido durante a determinação de $\mathbb{O}_{\infty}$.

Sejam $\hat{Y}$ e $\hat{U}$ vetores com os valores preditos das variáveis de saída e controle definidos como

$$
\hat{Y}=\left[\begin{array}{c}
\hat{y}(k+1 \mid k) \\
\vdots \\
\hat{y}\left(k+N+t^{*} \mid k\right)
\end{array}\right], \hat{U}=\left[\begin{array}{c}
\hat{u}(k \mid k) \\
\vdots \\
\hat{u}\left(k+N+t^{*} \mid k\right)
\end{array}\right] .
$$

Tendo em vista as relações (9) - (13), as equações de predição para $\hat{Y}$ e $\hat{U}$ podem ser escritas na forma

$$
\begin{gathered}
\hat{Y}=H \hat{V}+\Phi x(k), \\
\hat{U}=H_{U} \hat{V}+\Phi_{U} x(k),
\end{gathered}
$$

sendo $H \in \mathbb{R}^{q\left(N+t^{*}\right) \times p N}, H_{u} \in \mathbb{R}^{p\left(N+t^{*}+1\right) \times p N}, \Phi \in$ $\mathbb{R}^{q\left(N+t^{*}\right) \times n}, \Phi_{U} \in \mathbb{R}^{p\left(N+t^{*}+1\right) \times n}$ matrizes construídas a partir de $A, B, C$ e $K$ (Maciejowski, 2002).

Assim, dadas restrições da forma

$$
\begin{aligned}
& u_{P, \min }-u_{\text {ref }} \leq \hat{u}(k+i \mid k) \leq u_{P, \max }-u_{r e f}, \\
& i=0,1, \ldots, N+t^{*} \\
& y_{P, \min }-C x_{r e f} \leq \hat{y}(k+i \mid k) \leq y_{P, \max }-C x_{r e f}, \\
& i=1,2, \ldots, N+t^{*}
\end{aligned}
$$

e lembrando que $x(k)=x_{P}(k)-x_{\text {ref }}$ a minimização da função de custo (18) fica sujeita ao seguinte conjunto de desigualdades lineares:

$$
\begin{aligned}
& {\left[\begin{array}{c}
H_{U} \\
-H_{U} \\
H \\
-H
\end{array}\right] \hat{V} \leq} \\
& {\left[\begin{array}{c}
{\left[u_{P, \max }-u_{r e f}\right]_{N+t^{*}+1}-\Phi_{U}\left(x_{P}(k)-x_{r e f}\right)} \\
\Phi_{U}\left(x_{P}(k)-x_{r e f}\right)-\left[u_{P, \min }-u_{r e f}\right]_{N+t^{*}+1} \\
{\left[y_{P, \max }-C x_{r e f}\right]_{N+t^{*}}-\Phi\left(x_{P}(k)-x_{r e f}\right)} \\
\Phi\left(x_{P}(k)-x_{r e f}\right)-\left[y_{P, \min }-C x_{r e f}\right]_{N+t^{*}}
\end{array}\right],}
\end{aligned}
$$

em que $[\bullet]_{m}$ é um operador que empilha $m$ cópias de uma dado vetor coluna •. Portanto, o problema de otimização 
pode ser resolvido empregando algoritmos de Programação Quadrática (Maciejowski, 2002), pois há número finito de restrições lineares e o custo dado pela Eq. (18) é quadrático.

\section{GERENCIAMENTO DE REFERÊNCIA E RELAXAMENTO DE RESTRIÇÃO}

A formulação de controle preditivo descrita na Seção 2 garante factibilidade recursiva do problema de otimização e estabilidade da malha de controle (Rossiter, 2006), desde que as restrições (25) sejam inicialmente factíveis e os limites sobre $u$ e $y$ não sejam subsequentemente alterados. Neste trabalho, serão tratados problemas de não-factibilidade devido às seguintes causas:

1. Não-factibilidade inicial. Como as restrições em (25) dependem da diferença $x_{P}(k)-x_{r e f}$, o problema de otimização pode não ser factível no instante inicial $k=$ 0 se o estado $x_{P}(0)$ estiver muito distante da referência $x_{r e f}$.

2. Perda de factibilidade devido à ocorrência de falha de atuador. $O$ tipo de falha aqui considerado consiste em uma alteração nos limites físicos do controle, com aumento de $u_{P, \min }$ e/ou redução de $u_{P, \max }$. Assume-se que na prática tal alteração seria informada por um módulo de detecção e identificação de falhas. Desse modo, a partir da ocorrência da falha, a restrição (6) passaria a ser

$$
u_{f a l, \min }-u_{\text {ref }} \leq u \leq u_{f a l, \max }-u_{\text {ref }},
$$

sendo $u_{f a l, \min }$ e $u_{f a l, \max }$ os novos limites para a excursão de $u_{P}$. Com isso, o problema de otimização resultante pode se revelar não-factível.

Na abordagem aqui adotada, tais problemas serão tratados através do relaxamento de restrições operacionais sobre a saída da planta e/ou do gerenciamento da referência $x_{r e f}$.

No que diz respeito ao relaxamento de restrições, considerase que os limites de saída $y_{P, \min }, y_{P, \max }$ em (5) possam ser relaxados até limites físicos $y_{P, \min }^{f i s}, y_{P, \text { max }}^{f i s}$ préestabelecidos de modo a contornar o problema de nãofactibilidade. Para isso, serão introduzidas variáveis de folga $\epsilon_{s}, \epsilon_{i} \in \mathbb{R}^{q}$ em (7), passando-se a impor:

$$
\begin{gathered}
y_{P, \text { min }}-\epsilon_{i}-C x_{r e f} \leq y \leq y_{P, \max }+\epsilon_{s}-C x_{r e f}, \\
0 \leq \epsilon_{s} \leq y_{P, \text { max }}^{\text {fis }}-y_{P, \text { max }}, \\
0 \leq \epsilon_{i} \leq y_{P, \min }-y_{P, \text { min }}^{\text {fis }} .
\end{gathered}
$$

Já o gerenciamento da referência consiste em encontrar uma pseudo-referência

$$
x_{r e f}^{\prime}=x_{r e f}-\mu
$$

a cada instante $k$ a fim de tornar o problema de otimização factível e direcionar paulatinamente o sistema à referência original $x_{r e f}$. No caso geral, mudanças na referência $x_{r e f}$ também ocasionam alterações (denotadas por $\eta$ ) no valor de regime correspondente para o controle $\left(u_{r e f}\right)$, resultando na seguinte pseudo-referência para o controle:

$$
u_{\text {ref }}^{\prime}=u_{\text {ref }}-\eta \text {. }
$$

A relação entre $\mu$ e $\eta$ pode ser estabelecida da seguinte maneira. Em regime, a relação entre os valores de $x_{r e f}$ e $u_{r e f}$ obtida a partir da equação de estado da planta é:

$$
\left(I_{n}-A\right) x_{r e f}=B u_{r e f},
$$

sendo $I_{n}$ uma matriz identidade $n \times n$. Da mesma forma, a Eq. (32) precisa ser satisfeita pelos novos valores de regime $x_{r e f}^{\prime}$ e $u_{r e f}^{\prime}$, isto é,

$$
\left(I_{n}-A\right) x_{r e f}^{\prime}=B u_{r e f}^{\prime} .
$$

De (30), (31) e (33), tem-se que $\left(I_{n}-A\right)\left(x_{r e f}-\mu\right)=$ $B\left(u_{r e f}-\eta\right)$. Tendo em vista (32), conclui-se então que

$$
\left(I_{n}-A\right) \mu=B \eta .
$$

Por fim, substituindo $u_{r e f}$ por $u_{r e f}-\eta$ em (26) e $x_{r e f}$ por $x_{r e f}-\mu$ em (27), tais restrições passam a ser escritas como

$$
\begin{gathered}
u_{f a l, \text { min }}-u_{r e f}+\eta \leq u \leq u_{f a l, \text { max }}-u_{r e f}+\eta, \\
y_{P, \text { min }}-\epsilon_{i}-C x_{r e f}+C \mu \leq y \leq y_{P, \max }+\epsilon_{s}-C x_{r e f}+C \mu,
\end{gathered}
$$

estando as variáveis adicionais $\epsilon_{i}, \epsilon_{s}, \mu$ e $\eta$ sujeitas a (28), (29) e (34).

Vale salientar que estas alterações impõem a necessidade de uma nova determinação do MAS a cada vez que os valores de $\epsilon_{i}, \epsilon_{s}, \mu, \eta, u_{f a l, \min }$ ou $u_{f a l, \max }$ forem mudados. A abordagem proposta a seguir permite parametrizar o MAS em termos dos possíveis valores dessas variáveis, evitando assim a necessidade de se repetir em tempo real todo o procedimento envolvido na determinação de tal conjunto.

\subsection{Parametrização do MAS}

A parametrização do MAS pode ser feita através do uso de um estado aumentado $\bar{x}$ definido como

$$
\bar{x}=\left[\begin{array}{c}
x \\
\mu \\
\eta \\
\epsilon_{s} \\
\epsilon_{i} \\
u_{f a l, \max } \\
u_{f a l, \min }
\end{array}\right],
$$


que evolui dentro do MAS de acordo com

$$
\bar{x}(k+1)=\bar{A} \bar{x}(k), \quad \bar{A}=\left[\begin{array}{cc}
A-B K & 0 \\
0 & I_{n+2 q+3 p}
\end{array}\right] .
$$

Vale salientar que a matriz identidade $I_{n+2 q+3 p}$ multiplica as componentes adicionais do estado porque estas são supostas constantes ao longo do horizonte de predição. Embora $\bar{A}$ tenha autovalores na borda do círculo unitário (autovalores em +1 associados à matriz $I_{n+2 q+3 p}$ ), ainda é possível determinar o MAS em número finito de passos porque a dinâmica dada por (38) é estável no sentido de Lyapunov (Gilbert e Tan, 1991).

Para levar em conta as restrições (28), (29), (34), (35) e (36) pode-se definir uma variável de saída $\bar{z}$ como

$$
\bar{z}=\left[\begin{array}{c}
y-C \mu-\epsilon_{s} \\
-y+C \mu-\epsilon_{i} \\
\epsilon_{s} \\
\epsilon_{i} \\
u-\eta-u_{f a l, \max } \\
-u+\eta+u_{f a l, \min } \\
u_{f a l, \max } \\
u_{f a l, \min } \\
\left(I_{n}-A\right) \mu-B \eta
\end{array}\right]
$$

que estará sujeita às seguintes restrições:

$$
\left[\begin{array}{c}
-\infty \\
-\infty \\
0 \\
0 \\
-\infty \\
-\infty \\
u_{f a l, \max }^{\text {pior }} \\
u_{P, \min } \\
0
\end{array}\right] \leq \bar{z} \leq\left[\begin{array}{c}
y_{P, \max }-C x_{r e f} \\
C x_{r e f}-y_{P, \min } \\
y_{P, \max }^{\text {fis }}-y_{P, \max } \\
y_{P, \min }-y_{P, \min }^{f i s} \\
-u_{r e f} \\
u_{r e f} \\
u_{P, \max } \\
u_{f a l, \min }^{\text {pior }} \\
0
\end{array}\right]
$$

em que $u_{f a l, \text { min }}^{\text {pior }}$ e $u_{f a l, \text { max }}^{\text {pior }}$ correspondem aos valores de $u_{f a l, \min }$ e $u_{f a l, \max }$ no pior caso assumido para as possíveis falhas de atuador. A notação $-\infty$ empregada em (40) indica que a variável em questão não está limitada inferiormente. Vale ainda salientar que a última restrição em (40) corresponde à restrição de igualdade expressa em (34).
Lembrando que $u=-K x$ dentro do MAS, a função de saída para a determinação do MAS torna-se $\bar{z}=\bar{C} \bar{x}$ com

$$
\bar{C}=\left[\begin{array}{ccccccc}
C & -C & 0 & -I_{q} & 0 & 0 & 0 \\
-C & C & 0 & 0 & -I_{q} & 0 & 0 \\
0 & 0 & 0 & I_{q} & 0 & 0 & 0 \\
0 & 0 & 0 & 0 & I_{q} & 0 & 0 \\
-K & 0 & -I_{p} & 0 & 0 & -I_{p} & 0 \\
K & 0 & I_{p} & 0 & 0 & 0 & I_{p} \\
0 & 0 & 0 & 0 & 0 & I_{p} & 0 \\
0 & 0 & 0 & 0 & 0 & 0 & I_{p} \\
0 & \left(I_{n}-A\right) & -B & 0 & 0 & 0 & 0
\end{array}\right] .
$$

Tendo-se realizado a determinação do MAS associado à dinâmica (38) com o conjunto de restrições (40), pode-se particularizá-lo em tempo real fixando-se os valores de $\mu$, $\eta, \epsilon_{s}, \epsilon_{i}, u_{f a l, \max }$ e $u_{f a l, m i n}$. O conjunto $\overline{\mathbb{O}}_{\infty}$ assim obtido permanece invariante com respeito à trajetória do estado $x$, conforme mostrado no Apêndice B. Convém ressaltar que, como discutido anteriormente, a restrição terminal $\hat{x}(k+N \mid k) \in \overline{\mathbb{O}}_{\infty}$ equivale a impor as restrições sobre $\hat{u}(k+i \mid k)$ e $\hat{y}(k+i \mid k)$ até $i=N+\bar{t}^{*}$, sendo $\bar{t}^{*}$ obtido durante a determinação off-line do MAS parametrizado.

Deve-se ainda salientar que os valores de $u_{f a l, \max } \mathrm{e}$ $u_{f a l, \min }$ são iguais a $u_{P, \max }$ e $u_{P, \min }$, respectivamente, em condições normais de operação. Ao ocorrer uma falha, considera-se, por hipótese, que os novos valores de $u_{f a l, \max }$ e $u_{f a l, \text { min }}$ sejam informados ao controlador, de modo a adequar as restrições. Por outro lado, os valores de $\mu, \eta, \epsilon_{s}$ e $\epsilon_{i}$ são graus de liberdade adicionais que podem ser explorados pelo controlador preditivo, como detalhado a seguir.

\subsection{Formulação do problema de otimiza- ção}

Considerando o gerenciamento de referência e o relaxamento das restrições de saída, o problema de otimização a ser resolvido no instante $k$ passa a envolver $\hat{V}, \mu, \eta, \epsilon_{s}$ e $\epsilon_{i}$ como variáveis de decisão. Nesse caso, as restrições (25) sobre $\hat{V}$ devem ser modificadas para levar em conta a alteração de $x_{r e f}$ e $u_{r e f}$ por $x_{r e f}-\mu$ e $u_{r e f}-\eta$, bem como a introdução das variáveis de folga $\epsilon_{s}$ e $\epsilon_{i}$. Adicionalmente, os limites $u_{P, \max }$ e $u_{P, \min }$ passam a ser $u_{f a l, \max }$ e $u_{f a l, \min }$ e $t^{*}$ deve ser substituído por $\bar{t}^{*}$.

Com isso, o problema de otimização a ser resolvido no instante $k$ passa a ser

$\min _{\hat{V}, \mu, \eta, \epsilon_{s}, \epsilon_{i}} \hat{V}^{T} \Psi \hat{V}+\mu^{T} W_{\mu} \mu+\eta^{T} W_{\eta} \eta+\epsilon_{s}^{T} W_{\epsilon_{s}} \epsilon_{s}+\epsilon_{i}^{T} W_{\epsilon_{i}} \epsilon_{i}$, 
s.a.

$\left[\begin{array}{c}H_{U} \\ -H_{U} \\ H \\ -H\end{array}\right] \hat{V} \leq$

$\left[\begin{array}{c}{\left[u_{f a l, \max }-u_{r e f}+\eta\right]_{N+\bar{t}^{*}+1}-\Phi_{U}\left(x_{P}(k)-x_{r e f}+\mu\right)} \\ \Phi_{U}\left(x_{P}(k)-x_{r e f}+\mu\right)-\left[u_{f a l, m i n}-u_{r e f}+\eta\right]_{N+\bar{t}^{*}+1} \\ {\left[y_{P, \max }+\epsilon_{s}-C x_{r e f}+C \mu\right]_{N+\bar{t}^{*}}-\Phi\left(x_{P}(k)-x_{r e f}+\mu\right)} \\ \Phi\left(x_{P}(k)-x_{r e f}+\mu\right)-\left[y_{P, \text { min }}-\epsilon_{i}-C x_{r e f}+C \mu\right]_{N+\bar{t}^{*}}\end{array}\right]$

$(I-A) \mu-B \eta=0$,

$0 \leq \epsilon_{s} \leq y_{P, \max }^{\text {fis }}-y_{P, \max }$,

$0 \leq \epsilon_{i} \leq y_{P, \min }-y_{P, \min }^{\text {fis }}$,

em que $W_{\mu}, W_{\eta}, W_{\epsilon_{s}}$ e $W_{\epsilon_{i}}$ são matrizes positivo-definidas de pesos. Quanto maiores forem os pesos $W_{\mu}, W_{\eta}, W_{\epsilon_{s}}$ e $W_{\epsilon_{i}}$ em comparação com $\Psi$, mais a solução se aproxima da que seria obtida se não houvesse necessidade de gerenciamento de referência e/ou relaxamento de restrições. Ainda, visando priorizar o respeito às restrições operacionais sobre a saída, podem-se manipular $W_{\epsilon_{s}}$ e $W_{\epsilon_{i}}$ de forma que as parcelas a eles associadas no custo tenham maior magnitude do que aquelas associadas a $W_{\mu}$ e $W_{\eta}$. Com isso, prioriza-se o gerenciamento da referência como solução para o problema de não-factibilidade.

Tendo-se resolvido o problema de otimização (42), o controle a ser aplicado à planta é dado por

$$
u_{P}(k)=u_{r e f}-\eta^{*}+\hat{v}^{*}(k \mid k)-K\left(x_{P}(k)-x_{r e f}+\mu^{*}\right) .
$$

\section{RESULTADOS}

Para ilustrar o uso da estratégia proposta, empregou-se um modelo de simulação da forma $\dot{x}_{P}=A_{c} x_{P}+B_{c} u_{P}$, com

$$
A_{c}=\left[\begin{array}{cc}
-14 & -12,25 \\
8 & 0
\end{array}\right], \quad B_{c}=\left[\begin{array}{c}
0,25 \\
0
\end{array}\right]
$$

sendo o índice $c$ utilizado para salientar que se trata de uma equação de estado a tempo contínuo. A matriz $A_{c}$ possui autovalores $-7 \pm 7 \imath$, que correspondem a uma frequência natural $\omega_{n}=10 \mathrm{rad} / \mathrm{s}$. A fim de implementar o MPC, o modelo foi discretizado com período de amostragem de $0,04 s$, que é aproximadamente 15 vezes menor do que o período associado à frequência natural $\omega_{n}$.

A tarefa de controle a ser realizada consiste em conduzir o estado da planta para a origem (isto é, $x_{r e f}=0$ ), partindo de uma dada condição inicial. Considera-se que as variáveis de estado estejam sujeitas a limites operacionais $-2 \leq x_{P, 1}, x_{P, 2} \leq 2$, que podem ser relaxados até limites físicos $-2,5 \leq x_{P, 1}, x_{P, 2} \leq 2,5$ em caso de não-factibilidade.
Tais restrições são incluídas na estratégia proposta definindose que as variáveis de saída $y_{P}$ sujeitas a restrições sejam os próprios estados, isto é, $y_{P}=C x_{P} \operatorname{com} C=I_{2}$.

Adicionalmente, impõe-se que o controle $u_{P}$ deva permanecer na faixa $-0,1 \leq u_{P} \leq 0,1$ em condições normais de operação. Assume-se ainda que a ocorrência de falha no atuador restrinja a excursão do controle à faixa $-0,03 \leq u_{P} \leq 0,03$. Tal falha foi introduzida no instante $t_{f a l}=0,12 \mathrm{~s}$ (isto é, três períodos de amostragem após o início da simulação).

O controlador LQR utilizado na malha interna foi projetado com matrizes de peso $Q_{l q r}=10 I_{2}$ e $R_{l q r}=1$. O horizonte adotado no MPC foi $N=5$. Os pesos atribuídos às variáveis de relaxamento na função de custo (42) foram $W_{\mu}=I_{2}$, $W_{\eta}=1$ e $W_{\epsilon_{s}}=W_{\epsilon_{i}}=10^{3} \times I_{2}$.

As simulações foram realizadas a partir da condição inicial $x_{P}(0)=\left[\begin{array}{ll}-1,8 & -1,8\end{array}\right]^{T}$. Embora esta condição inicial se encontre dentro dos limites operacionais estabelecidos sobre os estados, o problema de otimização associado não se mostra factível, o que justifica o emprego da metodologia proposta. Como resultado, o problema de não-factibilidade inicial pôde ser contornado através da utilização conjunta dos procedimentos de relaxamento de restrições operacionais e gerenciamento de referência.

Conforme mostra a Fig. 2, a restrição operacional inferior de $x_{P, 2}$ foi relaxada durante os dois primeiros períodos de amostragem através do uso da variável de folga $\epsilon_{i}^{2}$. Nos instantes posteriores, $\epsilon_{i}^{2}$ permaneceu igual a zero, indicando que a restrição correspondente pôde ser mantida no seu valor original. Não houve necessidade de se relaxar a restrição operacional superior de $x_{P, 2}$, nem tampouco as restrições operacionais inferior e superior de $x_{P, 1}$ (isto é, as variáveis de folga $\epsilon_{s}^{2}, \epsilon_{i}^{1}$ e $\epsilon_{s}^{1}$ permaneceram iguais a zero durante toda a tarefa de controle).

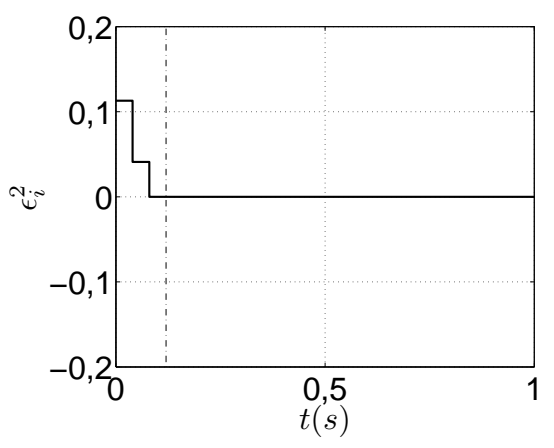

Figura 2: Evolução temporal da variável de folga $\epsilon_{i}^{2}$ associada à restrição operacional inferior do estado $x_{P, 2}$. A linha vertical tracejada indica o instante de ocorrência da falha. 
A Fig. 3 exibe o comportamento da pseudo-referência $\mu_{2}$ para o estado $x_{P, 2}$. Observa-se que foi realizado um gerenciamento da referência nos dois primeiros períodos de amostragem. Tal gerenciamento, em conjunto com o relaxamento de restrição acima descrito, foi realizado de modo a contornar a não-factibilidade inicial. Já no terceiro período de amostragem o gerenciamento da referência passou a ser desnecessário, o que indica que o controlador foi capaz de conduzir o estado $x_{P}$ ao domínio de atração associado à referência original. Contudo, com a introdução da falha em $t_{f a l}=0,12 \mathrm{~s}$, a faixa de valores admissíveis do controle foi reduzida, diminuindo assim o domínio de atração e causando novamente um problema de não-factibilidade. Assim, mais uma vez o gerenciamento da referência passou a ser necessário até o estado $x_{P}$ ingressar no novo domínio de atração, o que ocorreu no instante $t=0,40 s$.

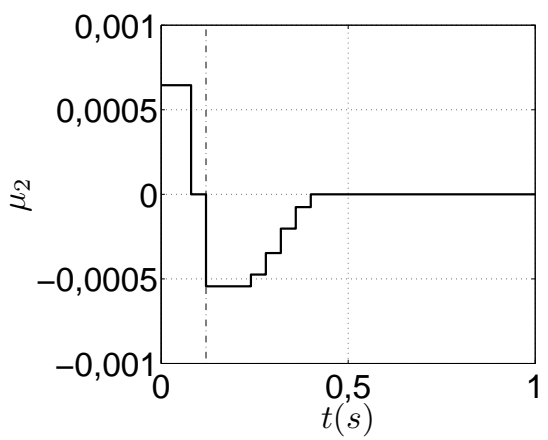

Figura 3: Evolução temporal da pseudo-referência $\mu_{2}$. A linha vertical tracejada indica o instante de ocorrência da falha.

Aliada ao gerenciamento da referência para o estado, ocorre a mudança na pseudo-referência do controle. Esta pode ser vista na Fig. 4. Nota-se que seu perfil é similar ao da pseudoreferência $\mu_{2}$, visto que ambas são relacionadas pelo vínculo algébrico (34).

Convém salientar que a pseudo-referência $\mu_{1}$ para o estado $x_{P, 1}$ permaneceu igual a zero durante o curso de toda a manobra, isto é, não foi necessário nenhum ajuste na referência do estado $x_{P, 1}$ para recuperar a factibilidade do problema de otimização. Da mesma forma, o relaxamento de restrições só se mostrou necessário durante os instantes iniciais, como já comentado com respeito à Fig. 2.



Figura 4: Evolução temporal da pseudo-referência de controle $\eta$. A linha vertical tracejada indica o instante de ocorrência da falha.

A Fig. 5 apresenta a evolução dos estados $x_{P, 1}$ e $x_{P, 2}$, mostrando que os mesmos foram conduzidos até a origem, como desejado. Vale notar que a restrição operacional inferior de $x_{P, 2}$ (correspondente à linha horizontal tracejada em -2) foi ultrapassada nos instantes iniciais, como resultado do relaxamento realizado. Contudo, as restrições físicas (correspondentes às linhas horizontais contínuas em $\pm 2,5$ na Fig. 5) foram respeitadas.

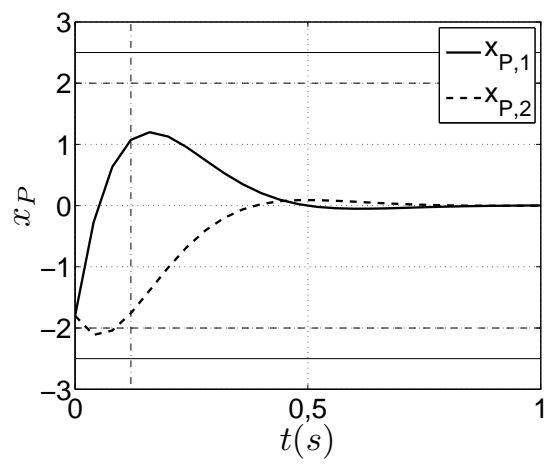

Figura 5: Evolução temporal dos estados. As linhas horizontais contínuas e tracejadas indicam os limites físicos e operacionais de $x_{P}$. A linha vertical tracejada indica o instante de ocorrência da falha.

Como se pode observar na Fig. 6, o sinal de controle $u_{P}$ foi mantido dentro da faixa de $\pm 0,1$ até a ocorrência da falha em $t_{f a l}=0,12 s$. A partir desse instante, a excursão do controle passou a ser limitada a $\pm 0,03$. 


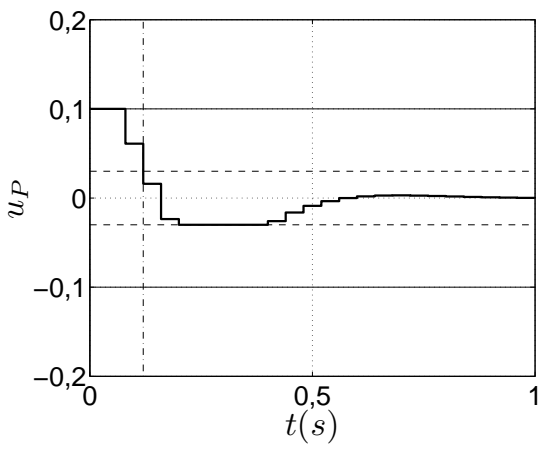

Figura 6: Evolução temporal do controle. As linhas horizontais contínuas e tracejadas indicam os limites sobre a excursão de $u_{P}$ em condições normais e na presença de falha, respectivamente. A linha vertical tracejada indica o instante de ocorrência da falha.

O tempo de computação a cada período de amostragem é apresentado na Fig. 7. Para obter esses dados a simulação foi executada 100 vezes em um computador pessoal com processador Pentium(R) Dual-Core E5400 com clock de 2,7 GHz e a média dos tempos de computação foi tomada. Esse procedimento visou eliminar flutuações devidas a interrupções do sistema operacional estranhas à simulação. O ambiente de simulação utilizado foi o Matlab ${ }^{\circledR}$ 7.1, empregando-se a função quadprog do Matlab Optimization Toolbox para a solução dos problemas de programação quadrática. Na aferição do tempo de computação, isolou-se o trecho de código relativo à otimização realizada por meio da função quadprog, de forma a não incluir o tempo gasto em rotinas alheias à obtenção do sinal de controle. Adicionalmente, desabilitou-se a exibição das mensagens relacionadas ao resultado da otimização na tela do Matlab, para evitar que o tempo de atualização da tela fosse indevidamente contabilizado como parte da computação. Nota-se na Fig. 7 que a necessidade de lidar com a não-factibilidade aumenta a carga computacional nos instantes iniciais e logo após a ocorrência da falha, devido à necessidade de se realizar o relaxamento de restrições e o gerenciamento de referência.

É também ilustrativo analisar os resultados obtidos ao se introduzir a falha mais tardiamente dentro da manobra realizada. Para isso, a simulação foi repetida com $t_{f a l}=0,16 \mathrm{~s}$ e $t_{f a l}=0,20 \mathrm{~s}$. Neste último caso, a falha não acarretou perda de factibilidade, uma vez que o estado já se encontrava suficientemente próximo da origem.

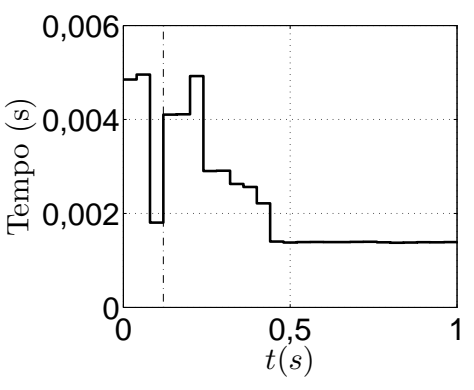

Figura 7: Evolução do tempo de computação do sinal de controle. A linha vertical tracejada indica o instante de ocorrência da falha.

A Fig. 8 mostra o valor da pseudo-referência $\mu_{2}$ para o estado $x_{P, 2}$ com a falha ocorrendo em $t_{f a l}=0,16 \mathrm{~s}$ e $t_{f a l}=0,20 \mathrm{~s}$. Percebe-se por meio de comparação com a Fig. 3 que, quanto mais tardiamente a falha ocorre, menor é a duração necessária do gerenciamento da referência, uma vez que o estado $x_{P}$ já se encontra mais perto da origem. Os valores da pseudoreferência de controle $\eta$ na Fig. 9 corroboram esta observação.

Os resultados obtidos para a evolução temporal da variável de folga $\epsilon_{i}^{2}$ e também do estado $x_{P}$ não estão apresentados por serem similares aos já vistos nas Figuras 2 e 5, respectivamente.

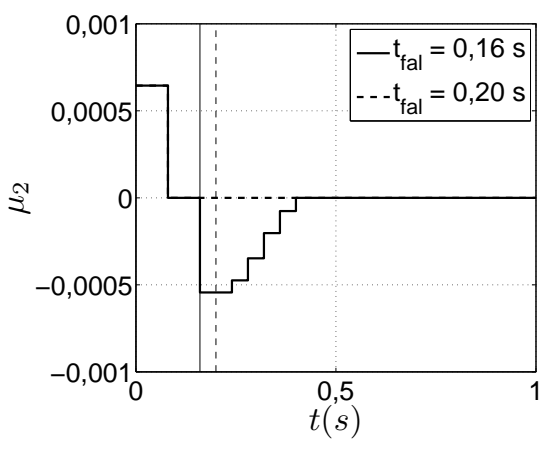

Figura 8: Evolução temporal da pseudo-referência $\mu_{2}$. As linhas verticais contínua e tracejada indicam a ocorrência de falha em $t_{f a l}=0,16 \mathrm{~s}$ e $t_{f a l}=0,20 \mathrm{~s}$, respectivamente. 


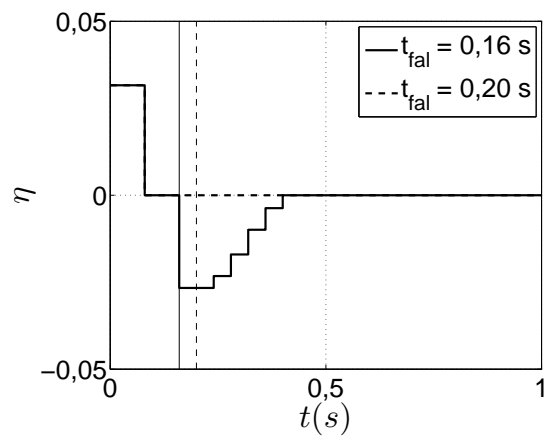

Figura 9: Evolução temporal da pseudo-referência de controle $\eta$. As linhas verticais contínua e tracejada indicam a ocorrência de falha em $t_{f a l}=0,16 \mathrm{~s}$ e $t_{f a l}=0,20 \mathrm{~s}$, respectivamente.

A Fig. 10 mostra o sinal de controle resultante. Pode-se notar que as restrições são adequadamente obedecidas, o que mais uma vez indica a capacidade de contornar os problemas advindos da falha.

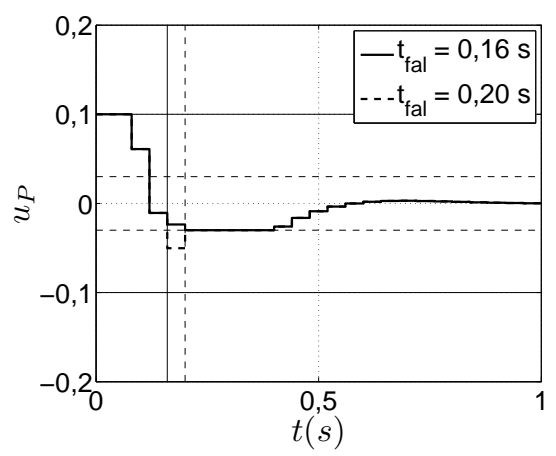

Figura 10: Evolução temporal do controle. As linhas horizontais contínuas e tracejadas indicam os limites sobre a excursão de $u_{P}$ em condições normais e na presença de falha, respectivamente. As linhas verticais contínua e tracejada indicam a ocorrência de falha em $t_{f a l}=0,16 \mathrm{~s}$ e $t_{f a l}=0,20 \mathrm{~s}$, respectivamente.

Os esforços computacionais demandados com a falha a partir de $t_{f a l}=0,16 s$ (linha contínua) e $t_{f a l}=0,20 s$ (linha tracejada) encontram-se na Fig. 11. Assim como na Fig. 7, os picos de carga computacional são observados no início da manobra, devido à necessidade de contornar a não-factibilidade inicial. Observa-se também um pico logo após a falha em $t_{f a l}=0,16 s$. Já com $t_{f a l}=0,20 s$, o aumento do tempo de computação após a falha foi menos expressivo. Isso pode ser explicado pelo fato de que, no último caso, mantém-se a factibilidade mesmo na presença da falha, não sendo necessário recorrer aos procedimentos de relaxamento de restrições ou gerenciamento de referência.

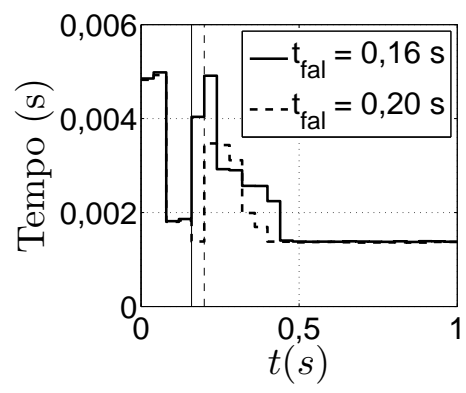

Figura 11: Evolução do tempo de computação do sinal de controle ao se introduzir a falha a partir de $t_{f a l}=0,16 \mathrm{~s}$ ou $t_{f a l}=0,20 s$.

\section{CONCLUSÕES}

Este artigo apresentou uma estratégia para o tratamento de problemas de não-factibilidade em controle preditivo através do relaxamento de restrições operacionais de saída e/ou gerenciamento da referência. Tal estratégia contempla casos de não-factibilidade inicial e também de perda de factibilidade devido a falhas de atuador que reduzam a excursão permitida para a variável de controle. Para isso, foi desenvolvido um procedimento de parametrização do conjunto invariante utilizado como restrição terminal no MPC. Com isso, evita-se a necessidade de se repetir a determinação de tal conjunto durante o tratamento da não-factibilidade. Como resultado, torna-se possível obter valores apropriados da pseudoreferência e do relaxamento de restrições de saída como resultado de um problema de programação quadrática.

Vale salientar que a abordagem proposta é voltada a problemas de regulação em torno de um ponto de equilíbrio. Esta característica é diretamente herdada de (Limon et al., 2008) e (Almeida e Leissling, 2010). Uma extensão para problemas de rastreamento poderia ser baseada na escolha de uma sequência de pontos de equilíbrio nas proximidades da trajetória considerada. Nesse caso, cada um dos pontos de equilíbrio seria uma referência intermediária para o controlador. A comutação de uma referência para a seguinte poderia ser realizada quando o problema de otimização envolvendo a nova referência se mostrasse factível. Esta ideia foi explorada em (Cavalca, 2011) e (Cavalca et al., 2012) no contexto de controle preditivo empregando desigualdades matriciais lineares. Em princípio, seria possível relaxar o requisito de que as referências intermediárias correspondam a pontos de equilíbrio. Isso impediria o uso de conjuntos terminais invariantes na forma adotada no presente trabalho, mas tal dificuldade poderia ser contornada empregando abordagens como a pro- 
posta em (Richards e How, 2006), que utiliza conjuntos-alvo não-invariantes. Essa possibilidade será explorada em trabalhos futuros.

Por simplicidade, o procedimento de relaxamento de restrições empregado neste trabalho envolveu apenas as variáveis de saída. Contudo, seria possível contemplar restrições operacionais de controle, considerando que as mesmas possam ser relaxadas até limites físicos mais amplos. Nesse caso, a falha de atuador acarretaria a imposição de limites mais estreitos sobre a variável de folga associada ao controle.

Investigações futuras podem incluir a realização de ensaios de bancada empregando a estratégia proposta. Seria também de valia desenvolver formas de parametrização do conjunto terminal para o tratamento de classes mais amplas de falhas, envolvendo alterações nos parâmetros ou mesmo na estrutura do modelo da planta.

\section{AGRADECIMENTOS}

Os autores agradecem o apoio da FAPESP (2009/12674-0 e 2006/58850-6) e CNPq (Produtividade em Pesquisa).

\section{APÊNDICE A}

Seja um sistema linear não-forçado com dinâmica descrita por uma equação de estado da forma

$$
x(k+1)=M x(k) .
$$

O estado inicial desse sistema é admissível quanto à saída com respeito a um conjunto de restrições $\mathbb{Y} \subset \mathbb{R}^{q}$ se a função de saída $z=h(x(x))$ satisfaz a condição $z(k) \in \mathbb{Y}, \forall k \geq$ 0 . O conjunto de todos os estados iniciais com esta propriedade é denominado MAS (Maximal Output Admissible Set), sendo usualmente simbolizado por $\mathbb{O}_{\infty}$. Tal conjunto é invariante no sentido de que $x(k) \in \mathbb{O}_{\infty} \Rightarrow x(k+1) \in \mathbb{O}_{\infty}$ (Gilbert e Tan, 1991).

Considerando uma função de saída linear (isto é, $z=C x$ ), e restrições da forma $f_{i}(z) \leq 0, i=1,2, \ldots, s$, um algoritmo para a determinação computacional do MAS pode ser encontrado em (Gilbert e Tan, 1991). Tal determinação equivale a impor o conjunto de restrições originais por um horizonte $t^{*} \geq 0$ finito, desde que o sistema seja estável no sentido de Lyapunov. Assim, mesmo no caso em que a dinâmica envolva integradores, pode-se caracterizar o conjunto invariante através de um número finito de restrições (González e Odloak, 2009).

\section{APÊNDICE B}

Seja um sistema linear não-forçado com dinâmica descrita por uma equação de estado da forma

$$
x(k+1)=M x(k),
$$

em que $x(k) \in \mathbb{R}^{n}$.

Seja ainda um estado aumentado $\bar{x}(k) \in \mathbb{R}^{n+m}$ definido como

$$
\bar{x}(k)=\left[\begin{array}{c}
x(k) \\
\theta(k)
\end{array}\right],
$$

em que $\theta(k) \in \mathbb{R}^{m}$ é um vetor constante de parâmetros, ou seja, $\theta(k+1)=\theta(k)$. Pode-se então escrever

$$
\bar{x}(k+1)=\bar{M} \bar{x}(k),
$$

com

$$
\bar{M}=\left[\begin{array}{cc}
M & 0 \\
0 & I_{m}
\end{array}\right] .
$$

Considere-se agora uma função de saída $\bar{z}$ definida como

$$
\bar{z}=\left[\begin{array}{c}
z_{x \theta} \\
z_{\theta}
\end{array}\right]=\left[\begin{array}{c}
h(x, \theta) \\
h_{\theta}(\theta)
\end{array}\right],
$$

estando $z_{x \theta}(k)=h(x(k), \theta(k))$ e $z_{\theta}(k)=h_{\theta}(\theta(k))$ sujeitas a restrições da forma

$$
\begin{gathered}
z_{x \theta}(k) \in \mathbb{Y}_{x \theta}, \\
z_{\theta}(k) \in \mathbb{Y}_{\theta} .
\end{gathered}
$$

Denote-se por $\overline{\mathbb{O}}_{\infty}$ o MAS associado à dinâmica (48) e às restrições (51), (52), isto é

$$
\overline{\mathbb{O}}_{\infty}=\left\{\bar{x}(0): z_{x \theta}(k) \in \mathbb{Y}_{x \theta}, z_{\theta}(k) \in \mathbb{Y}_{\theta}, \quad \forall k \geq 0\right\} .
$$

Seja agora $\theta_{c} \in \mathbb{R}^{m}$ um vetor fixado e admissível com respeito às restrições de $\theta$, isto é,

$$
h_{\theta}\left(\theta_{c}\right) \in \mathbb{Y}_{\theta} .
$$

Tem-se então que o conjunto $\mathbb{O}_{\infty}$ definido como

$$
\mathbb{O}_{\infty}=\left\{x(0):\left[\begin{array}{c}
x(0) \\
\theta_{c}
\end{array}\right] \in \overline{\mathbb{O}}_{\infty}\right\}
$$

é um MAS associado à dinâmica (46) e à restrição (51) particularizada para $\theta=\theta_{c}$.

\section{Demonstração:}

$\mathrm{O}$ conjunto $\mathbb{O}_{\infty}$ é admissível quanto à saída $z_{x \theta}(k)$ por construção. Com efeito, pela definição (55), se $x(0) \in \mathbb{O}_{\infty}$, temse que

$$
\left[\begin{array}{c}
x(0) \\
\theta_{c}
\end{array}\right] \in \overline{\mathbb{O}}_{\infty}
$$


Portanto, pela definição (53) a restrição (51) será respeitada $\forall k \geq 0$. Resta ainda demonstrar que $\mathbb{O}_{\infty}$ é o máximo conjunto admissível quanto à saída $z_{x \theta}(k)$.

Por absurdo, suponha que $\exists \xi \in \mathbb{R}^{n}$ tal que

$$
\begin{gathered}
x(0)=\xi \Rightarrow h\left(x(k), \theta_{c}\right) \in \mathbb{Y}_{x \theta}, \forall k \geq 0, \\
\xi \notin \mathbb{O}_{\infty} .
\end{gathered}
$$

Sabendo que $\theta_{c}$ satisfaz (54), tem-se que $z_{\theta}(k) \in \mathbb{Y}_{\theta}, \forall k \geq$ 0 . Por outro lado, $h\left(x(k), \theta_{c}\right) \in \mathbb{Y}_{x \theta}, \forall k \geq 0$, implica que $z_{x \theta}(k) \in \mathbb{Y}_{x \theta}, \forall k \geq 0$. Portanto, tem-se que $\left[\begin{array}{c}\xi \\ \theta_{c}\end{array}\right] \in \overline{\mathbb{O}}_{\infty}$. De (55), segue então que $\xi \in \mathbb{O}_{\infty}$, o que contradiz (57). Assim, conclui-se que todas as condições iniciais $\xi \in \mathbb{R}^{n}$ que satisfazem (56) devem pertencer a $\mathbb{O}_{\infty}$, mostrando que $\mathbb{O}_{\infty}$ é o máximo conjunto admissível quanto à saída $z_{x \theta}(k)$.

\section{REFERÊNCIAS}

Afonso, R. J. M. e Galvão, R. K. H. (2010). Controle preditivo com garantia de estabilidade nominal aplicado a um helicóptero com três graus de liberdade empregando relaxamento de restrições de saída, Proc. XVIII Congresso Brasileiro de Automática, pp. 1797 - 1804.

Afonso, R. J. M. e Galvão, R. K. H. (2010b). Predictive control of a helicopter model with tolerance to actuator faults, Proc. Conference on Control and Fault-Tolerant Systems (SysTol), pp. $744-751$.

Afonso, R. J. M. e Galvão, R. K. H. (2012). Infeasibility handling in constrained MPC. In: Zheng, T. (ed.) Frontiers of Model Predictive Control, Intech, Rijeka, Croatia, pp. $47-64$.

Almeida, F. A. e Leissling, D. (2010). Fault-tolerant model predictive control with flight-test results, Journal of Guidance, Control, and Dynamics 33(2): 363 - 375.

Alvarez, T. e de Prada, C. (1997). Handling infeasibilities in predictive control, Computers \& Chemical Engineering 21: S577 - S582.

Bemporad, A., Casavola, A. e Mosca (1997). Nonlinear control of constrained linear systems via predictive reference management, IEEE Trans. Automatic Control 42(3): $340-349$.

Bemporad, A. e Mosca (1994). Constraint fulfilment in feedback control via predictive reference management, Proc. 3rd IEEE Conf. Control Applications, Glasgow, UK, pp. $1909-1914$.

Camacho, E. F. e Bordons, C. (1999). Model Predictive Control, Springer-Verlag, London.
Cavalca, M. S. M. (2011). Controle Preditivo Reconfigurável para Acomodação de Falhas, Tese de Doutorado, ITA, São José dos Campos.

Cavalca, M. S. M., Galvão, R. K. H. e Yoneyama, T. (2012). Robust model predictive control using linear inequalities for the treatment of asymmetric output constraints, Journal of Control Science and Engineering (485784).

Chisci, L., Rossiter, J. A. e Zappa, G. (2001). Systems with persistent disturbances: predictive control with restricted constraints, Automatica 37(7): 1019 - 1028.

Cutler, C. R. e Ramaker, B. L. (1980). Dynamic matrix control - a computer control algorithm, Proc. Joint American Control Conference, IEEE, San Francisco.

Gilbert, E. G. e Kolmanovsky, I. (1995). Discrete-time reference governors for systems with state and control constraints and disturbance inputs, Proc. 34th IEEE Conference on Decision and Control.

Gilbert, E. G. e Tan, K. T. (1991). Linear systems with state and control constraints: the theory and application of maximal output admissible sets, IEEE Trans. Automatic Control 36(9): 1008 - 1020.

González, A. H. e Odloak, D. (2009). Enlarging the domain of attraction of stable mpc controllers, maintaining the output performance, Automatica 45: 1080 - 1085.

Kapasouris, P., Athans, M. e Stein, G. (1988). Design of feedback control systems for stable plants with saturating actuators, Proc. 27th IEEE Conference on Decision and Control.

Keviczky, T. e Balas, G. J. (2006). Receding horizon control of an F-16 aircraft: a comparative study, Control Engineering Practice 14(9): 1023 - 1033.

Kouvaritakis, B., Rossiter, J. A. e Cannon, M. (1998). Linear quadratic feasible predictive control, Automatica 34(12): 1583 - 1592 .

Limon, D., Alvarado, I., Alamo, T. e Camacho, E. (2008). MPC for tracking piecewise constant references for constrained linear systems, Automatica 44(9): 2382 2387.

Lopes, R. V. (2007). Modelagem e controle preditivo de um helicóptero com três graus de liberdade, Tese de mestrado, ITA, São José dos Campos.

Maciejowski, J. M. (2002). Predictive Control with Constraints, Prentice Hall, Harlow, England. 
Montandon, A. G., Borges, R. M. e Henrique, H. M. (2008). Experimental application of a neural constrained model predictive controller based on reference system, Latin American Applied Research 38: 51 - 62.

Normey-Rico, J. E. (2007). Controle preditivo baseado em modelo. In: Aguirre, L. A. (ed.) Enciclopédia de Automática, Blucher, São Paulo, Vol. 2, Cap. 11, pp. $242-$ 260.

Qin, S. J. e Badgwell, T. A. (2003). A survey of industrial model predictive control technology, Control Engineering Practice 11(7): 733 - 764.

Rawlings, J. B. e Muske, K. R. (1993). The stability of constrained receding horizon control, IEEE Trans. Automatic Control 38(10): 1512 - 1516.

Richards, A. e How, J. P. (2006). Robust variable horizon model predictive control for vehicle maneuvering, International Journal of Robust and Nonlinear Control 16: $333-351$.

Rodrigues, M. A. e Odloak, D. (2005). Robust MPC for systems with output feedback and input saturation, Journal of Process Control 15: 837 - 846.

Rossiter, J. A. (2003). Model-based Predictive Control: a practical approach, CRC Press, Boca Raton.

Rossiter, J. A. (2006). A global approach to feasilbility in linear MPC, Proc. UKACC international Control Conference, UKACC, Glasgow.

Scokaert, P. O. M. (1994). Constrained Predictive Control, Tese de doutorado, Univ. Oxford, UK.

Scokaert, P. O. M. e Rawlings, J. B. (1998). Constrained linear quadratic regulation, IEEE Trans. Automatic Control 43(8): 1163 - 1169.

Scokaert, P. e Rawlings, J. (1997). On infeasibilities in model predictive control, AIChE Symposium Series, pp. 331 334.

Vada, J., Slupphaug, O. e Johansen, T. A. (2001). Optimal prioritized infeasibility handling in model predictive control: parametric preemptive multiobjective linear programming approach, Journal of optimization theory and applications 109(2): $385-413$.

Vada, J., Slupphaug, O., Johansen, T. e Foss, B. (2001). Linear MPC with optimal prioritized infeasibility handling: application, computational issues and stability, Automatica 37(11): 1835 - 1843.

Zafiriou, E. e Chiou, H. (1993). Output constraint softening for SISO model predictive control, Proc. American Control Conference. 